\title{
Novel Serum Biomarkers Detected by Protein Array in Polycystic Ovary Syndrome with Low Progesterone Level
}

\author{
Qin Zheng ${ }^{\mathrm{a}}$ Feifei Zhou ${ }^{\mathrm{b}} \quad$ Xinyuan Cui ${ }^{\mathrm{a}}$ Mulin Liuc ${ }^{\mathrm{c}}$ Yulin Lia ${ }^{\mathrm{a}}$ Shuai Liu \\ Jichun Tan ${ }^{\mathrm{b}}$ Qiu Yan
}

aDepartment of Biochemistry and Molecular Biology, Dalian Medical University, Liaoning Provincial Core Lab of Glycobiology and Glycoengineering, Dalian, ${ }^{b}$ Reproduction Medicine Center, Obestetrics and Gynecology Department of Shengjing Hospital Affiliated to China Medical University, Shenyang, ${ }^{c}$ Clinical Laboratory Diagnostics, The First Affiliated Hospital of Dalian Medical University, Dalian, China

\section{Key Words}

PCOS • Progesterone • EREG • Inhibin $\beta A$

\begin{abstract}
Background/Aims: Polycystic ovary syndrome (PCOS), characterized by female infertility and metabolic abnormalities, is one of the most common endocrine disorders. The etiology of PCOS remains unknown. The comprehensive analysis of protein alterations in PCOS patients is meaningful for identifying diagnostic biomarkers of PCOS. Here, we explored the clinical value of serum proteins as novel biomarkers to detect PCOS with low progesterone level. Methods: A total of 43 patients with PCOS and 30 healthy women were enrolled. Protein array was used to detect the variations of serum proteins between PCOS patients and healthy women. The level of five serum proteins was further confirmed by ELISA and western blot. The human ovarian granulosa cells (KGN) was cultured to examine the underlying mechanism of PCOS. CCK8 assay and western blot were carried out to evaluate the alterations in proliferative ability, TUNEL assay and DAPI staining to detect the apoptosis of KGN cells. Results: Among the 507 proteins, we identified 76 differentially expressed serum proteins ( $\geqq 1.5$ fold), with 40 elevated and 36 decreased proteins. Moreover, 47 proteins were newly reported in PCOS. The alterations in the five significantly decreased proteins (EREG, inhibin $\beta A$, IDE, PDGF-D and KNG1) were further confirmed by ELISA and western blot. The level of these proteins were directly associated with the low progesterone, and the expression could be upregulated by progesterone. EREG and inhibin $\beta A$ also promoted the proliferation and inhibited the apoptosis of ovarian granulosa cells. Conclusion: The study highlights that serum proteins are differentially expressed in PCOS patients and healthy women, and EREG and inhibin $\beta A$ levels are upregulated by progesterone, which are correlated with ovarian functions. The study suggests that EREG and inhibin $\beta A$ may be applied as novel potential biomarkers for PCOS with low progesterone level.

Q. Zheng and F. Zhou contributed equally to this work.

Qiu Yan, Professor and Jichun Tan, Professor

\section{KARGER}

Department of Biochemistry and Molecular Biology of Dalian Medical University Dalian 116044, (China); Reproduction Medicine Center of Shengjing Hospital Affiliated to China Medical University, Shenyang 110004, (China)

E-Mail yanqiu63@126.com / tjczjh@163.com
\end{abstract}




\section{Cellular Physiology Cell Physiol Biochem 2018;46:2297-2310 \begin{tabular}{l|l} 
DOI: 10.1159/000489619 & a 2018 The Author(s). Published by S. Karger AG, Basel \\
www.karger.com/cpb
\end{tabular} Zheng et al.: Novel Serum Biomarkers for PCOS}

\section{Introduction}

Polycystic ovary syndrome (PCOS) is a complicated endocrine abnormality in women with childbearing age that is characterized by female infertility and metabolic aberrations [1]. Its incidence has increased yearly in reproductive age women, which is reported to be as high as $5-10 \%$ [2]. At present, PCOS is predominantly diagnosed by ultrasound scans of ovaries, clinical manifestations, and measurement of hormones in serum [3]. The etiology of PCOS is complex and remains uncertain. Numerous studies have shown that many factors are associated with PCOS. Therefore, it is imperative to discover diagnostic biomarkers to provide auxiliary indexes for PCOS detection.

As the main mediators, hormones are required for follicle maturation, ovulation, embryo development, and the establishment of endometrial receptivity [4, 5]. Hormones that regulate ovarian functions include luteinizing hormone (LH), follicle stimulating hormone (FSH), progesterone, estrogen and androgen. Progesterone is a steroid hormone produced by ovarian granulosa cells under the regulation of hypothalamus gonadotropin and pituitary LH [6]. LH promotes the formation of the corpus luteum after ovulation and stimulates the synthesis of progesterone [7]. In addition, progesterone can regulate ovarian functions and affect downstream target organs, such as uterus, oviduct and placenta [8]. Patients of PCOS are unable to form corpus luteum and generate low level of progesterone, thus leading to the dysfunction in ovulation [9]. Progesterone is also an essential pregnancy hormone in maintaining embryo development and endometrial receptivity [10]. Low dose progesterone has been reported to treat patients with infertility or possible abortion caused by ovulation dysfunction [11]. In pregnant patients with PCOS, appropriate progesterone treatment could reduce the occurrence of miscarriage, preeclampsia and premature delivery [12]. Therefore, the measurement of altered proteins caused by abnormal progesterone is indispensable for understanding the pathogenesis, and provides biomarkers for PCOS detection and treatment.

A variety of pathological processes are involved in the pathogenesis of PCOS, such as chronic inflammation, dysfunction of extracellular matrix degradation and proliferation or apoptosis of granulosa cells. The inhibition of granulosa cells proliferation leads to the impairment of follicle development, as well as follicular atresia and even ovulation disorder [13]. Furthermore, this process is also accompanied by changes in many molecules, like cytokines, growth factors and adhesion molecules. Recently, cytokines have emerged as key contributors to human reproductive activities, and participate in the modulation of ovarian functions [14]. Xia et al. found that IL-1 $\beta$ was increased in serum, and positively correlated with the incidence of PCOS [15]. Gallinelli et al. reported that IL-12 was decreased in the follicular fluid of PCOS patients compared to controls, and the increased IL-13 was associated with the occurrence and development of PCOS [16]. Moreover, growth factors and their receptors are also involved in regulating reproductive processes of the ovary. Epiregulin (EREG), a member of the EGF family, has been reported to promote oocyte maturation, ovulation and embryo implantation [17]. However, the role of EREG in PCOS has not yet been investigated. Inhibins belong to the superfamily of TGF $\beta$, and express in the ovary, testis, placenta and adrenal gland. They are produced and secreted by ovarian granulosa cells [18]. Inhibin $\beta A$ is one subunit of inhibin $A$, which is consist of an a subunit dislphide linked to a $\beta$ A subunit. Thus, the detection of inhibin $\beta A$ in serum may reflect the development of ovarian granulosa cells and follicles.

The serological measurement for disease detection is widely used clinically, as it is a nontraumatic and noninvasive method. Meanwhile, serum samples are relatively easy to obtain. Serological testing for PCOS includes six hormones: LH, FSH, progesterone, estrogen, androgen and prolactin [19]. The combination with other serum biomarkers may increase the accuracy and specificity of PCOS detection. In our study, we collected serum samples from PCOS patients with low progesterone level, and screened differentially expressed proteins by using protein array. We found five decreased proteins: EREG, inhibin $\beta$ A, insulysin (IDE), platelet-derived growth factor-D (PDGF-D) and kininogen (KNG1), and further explored the 
regulatory role of progesterone on the expression of these molecules, as well as the effects of EREG and inhibin $\beta A$ on the proliferation and apoptosis of ovarian granulosa cells.

\section{Materials and Methods}

\section{Ethics statement}

The clinical serum samples obtained got the approval of Clinical Ethics Review Board of Shengjing Hospital of China Medical University. The research was in accordance with Institutional Review Board of Dalian Medical University.

\section{Clinical samples}

The serum samples from 43 patients diagnosed with PCOS and 30 healthy women paired by BMI and age were collected from Shengjing Hospital of China Medical University. The diagnosis criteria was followed Rotterdam criteria of 2003: oligoovulation; clinical evidence of hyperandrogenism and/or hyperandrogenemia; PCO morphology (12 or more with 2-9 $\mathrm{mm}$ follicles, or $>10 \mathrm{ml}$ in volume in at least one ovary); Moreover, the exclusion of related disorders including congenital adrenal hyperplasia, Cushing's syndrome and androgen-producing tumors. The healthy women were with regular menstrual cycles and normal hormone levels, without family history of endocrine abnormality or evidence of PCOS, excessive acne or hirsutism. No subjects had used pharmacological treatment. Informed consent was given to every patient.

\section{Protein array}

Protein array was purchased from RayBiotech (Arraystar, Rockville, USA). Total protein was extracted and the protein concentration was determined by BCA protein assay kit (KANGCHEN, Shanghai, China). The protein array membranes were blocked in blocking buffer for $30 \mathrm{~min}$, and then incubated with samples at $4^{\circ} \mathrm{C}$ overnight. The samples were decanted and washed with washing buffer. After washing, the membranes were incubated with diluted biotin-conjugated antibodies for $1-2 \mathrm{~h}$ at room temperature. Then, the membranes were reacted with streptavidin- conjugated fluor, and scanned by a Axon GenePix laser scanner after washing thoroughly. The relative expression of proteins was made by comparing the signal intensities. Raw intensities were revised by background and normalized by median background signals. The intensities of signals were quantified by densitometry, and relative differences in expression levels of each sample were determined. Any $\geqq 1.5$ fold increase or $\leqq 0.65$ fold decrease in signal intensity for a single analyte between samples may be considered a measurable and significant difference in expression according to our experimental condition, which provided that both sets of signals were well above background (mean background +2 standard deviations, accuracy $\approx 95 \%$ ). Thus, for protein quantitation from protein array, the ratios of samples were weighted and normalized to control samples. For quantitative fold changes, we set a fold change $\geqq 1.5$ as cutoff for differentially expressed proteins selection.

\section{Cell culture}

A human ovarian granulosa cell line (KGN) was obtained from RIKEN (Tokyo, Japan). The KGN cells were undifferentiated which maintained the physiological characteristics of human ovarian granulosa cells. KGN cells were cultured in DMEM/F12 medium (Invitrogen, Grand Island, NY, USA) with 10\% fetal bovine serum, $100 \mathrm{U} / \mathrm{mL}$ penicillin-streptomycin and grown at $37^{\circ} \mathrm{C}, 5 \% \mathrm{CO}_{2}$ atmosphere.

\section{ELISA}

The inhibin $\beta$ A (Arraystar, Rockville, USA), EREG, IDE, PDGF-D and KNG1 (Elabscience, Wuhan, China) levels in serum and culture medium of KGN cells were determined by ELISA, and all of the steps were performed according to the manufacturer's instructions. The KGN cells were treated with progesterone for different concentrations $\left(0,10^{-6}, 10^{-5}\right.$ and $\left.10^{-4} \mathrm{M}\right)$ and different times $(0,24,48$ and $72 \mathrm{~h})$, and the culture medium were collected for ELISA assay. The samples $(100 \mu \mathrm{l})$ were coated in the plates at $37^{\circ} \mathrm{C}$ for $2.5 \mathrm{~h}$. The plates were then washed and blocked for $1 \mathrm{~h}$. Following washing, $100 \mu \mathrm{l}$ of the biotinylated human inhibin $\beta$ A, EREG, IDE, PDGF-D and KNG1 antibodies were added and incubated for another $45 \mathrm{~min}$ at $37^{\circ} \mathrm{C}$. Streptavidin-horseradish peroxidase conjugates $(100 \mu \mathrm{l})$ were added for $45 \mathrm{~min}$. The absorbance value was 


\section{Cellular Physiology Cell Physiol Biochem 2018;46:2297-2310

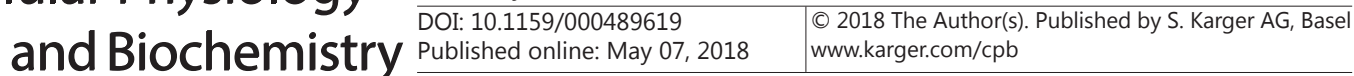 \\ Zheng et al.: Novel Serum Biomarkers for PCOS}

read and recorded at $450 \mathrm{~nm}$ after washing and colored. The concentration of inhibin $\beta A$, EREG, IDE, PDGF-D and KNG1 was measured according to the standard curve.

\section{Western blot}

Cells were lysed on ice for 20 min in RIPA lysis buffer containing protease inhibitor. The protein concentration was detected, and the extract was separated in a 12\% SDS-PAGE gel and transferred onto nitrocellulose membranes (Millipore, Billerica, MA, USA). After blocking with 5\% non-fat dry milk, the membranes were incubated with primary antibodies at $4^{\circ} \mathrm{C}$ overnight with anti-EREG (1:500, Abcam, Cambridge, UK), anti-inhibin $\beta$ A (1:500, Abcam, Cambridge, UK), anti-PDGF-D (1:500, Proteintech Group, Wuhan, China), anti-IDE (1:1000, Proteintech Group, Wuhan, China), anti-KNG1 (1:500, Proteintech Group, Wuhan, China), anti-PCNA (1:2000, Proteintech Group, Wuhan, China), anti-Bcl-2 (1:2000, Proteintech Group, Wuhan, China), anti-Bax (1:2000, Proteintech Group, Wuhan, China), anti-caspase 8 (1:500, Proteintech Group, Wuhan, China), anti-caspase 9 (1:500, Proteintech Group, Wuhan, China) and antiGAPDH (1:2000, Proteintech Group, Wuhan, China). Washing with TBST for three times, membranes were incubated with horse-radish peroxidase-conjugated goat anti-rabbit antibody (1:2000, ZSGB-BIO, Beijing, China) for $45 \mathrm{~min}$. GAPDH and Coomassie brilliant blue (CBB) was used as an equal loading control. The signals were visualized with enhanced chemiluminescence system, and the protein bands were analyzed with Image J software (NIH, Bethesda, MD, USA).

\section{Cell proliferation assay}

Cells were seeded in 96-well plate with a density of 3000 cells per well, and incubated with different concentrations of EREG $(0,10,100 \mu \mathrm{g} / \mathrm{ml}$, PeproTech, NJ, USA), inhibin $\beta A(0,10,100 \mu \mathrm{g} / \mathrm{ml}$, Abcam, Cambridge, UK) or their specific antibodies (anti-EREG: 1:200, Abcam, Cambridge, UK; anti-inhibin $\beta A$ : 1:100, Abcam, Cambridge, UK), respectively. The effects of EREG and inhibin $\beta A$ on cell proliferation were determined with Cell Counting Kit 8 (CCK8, Beyotime Biotechnology, Beijing, China) according to the protocols. CCK8 solution was added to the medium and incubated for $2 \mathrm{~h}$ at $37^{\circ} \mathrm{C}$. The absorbance at 450 $\mathrm{nm}$ was detected, and the data were recorded and analyzed. Triplicate independent experiments were performed.

\section{Immunofluorescent staining}

KGN cells were cultured on glass coverslip and fixed in 4\% para-formaldehyde for $20 \mathrm{~min}$ at room temperature, followed with permeabilized in $0.1 \%$ Triton X-100 for $10 \mathrm{~min}$. The primary antibody of PCNA (1:50) was used at $4^{\circ} \mathrm{C}$ overnight after blocking in $3 \%$ bovine serum for $1 \mathrm{~h}$. After washing with PBS for three times, cells were incubated with TRITC-conjugated goat anti-rabbit IgG (1:100, Sigma-Aldrich, St.Louis, MO, USA) for $1 \mathrm{~h}$ at room temperature. The nucleus was stained with DAPI (1:1000, Beyotime Biotechnology, Beijing, China) in dark for 5 min. Images were taken with a fluorescence microscope (Olympus, Tokyo, Japan), and the representative pictures were shown.

\section{Cell apoptosis assay}

Morphological evaluation of cell apoptosis was carried out by using DAPI staining. Cells grown on the coverslip after treatment were fixed with $4 \%$ para-formaldehyde for $20 \mathrm{~min}$ at room temperature, and incubated with $0.1 \%$ Triton X-100 for $10 \mathrm{~min}$. After washing, cells were stained with DAPI for 5 min in dark. Images were acquired under a fluorescence microscope.

\section{Statistical analysis}

The results obtained from at least three independent experiments were expressed as mean \pm S.E.M. Statistical analysis was carried out using the Prism version 6.0 program (GraphPad). Differences were analyzed with Student's $t$-test for comparison differences between groups by using SPSS 17.0 (SPSS, Inc, Chicago, IL). Spearman's correlation analysis was used to evaluate the relationship between progesterone and EREG, inhibin $\beta A$, IDE, PDGF-D and KNG1 in serum of patients with PCOS. $P$-value $<0.05$ was considered as statistical significance. 


\section{Cellular Physiology Cell Physiol Biochem 2018;46:2297-2310 and Biochemistry \begin{tabular}{c|c} 
DOI: 10.1159/000489619 \\
Published online: TVay 07, 2018 & $\begin{array}{l}\text { O 2018 The Author(s). Published by S. Karger AG, Basel } \\
\text { www.karger.com/cpb }\end{array}$
\end{tabular} Zheng et al.: Novel Serum Biomarkers for PCOS}

\section{Results}

Differential expression of proteins in serum of PCOS patients

To determine the alterations of serum proteins, protein array was used for screening. As shown, 507 proteins in serum were detected. Expression level of 76 proteins (Fig. 1) were changed over 1.5 times in serum of PCOS patients compared to healthy controls, and 47 kinds of proteins were newly reported. The results showed that there were 40 kinds of proteins with elevated level among the 507 proteins (Table 1), including growth factors (IGF-1, IGFBP-1, etc); angiogenesis factors (angiogenin, angiopoietin-2, etc); interleukins (IL-2 R $\alpha$, IL-29, etc); chemokines (CXCR4, CXCR6, etc); members of tumor necrosis factor family (TNF- $\alpha$, TNFRSF6, etc). Meanwhile, there were 36 proteins with reduced expression (Table 2), including growth factors (EREG, inhibin $\beta A$, etc); angiogenesis factors (PDGF-D, VEGF R3, etc); interleukins (IL-1 R4, IL-11, etc); chemokines (CCR5, CCR6, etc); members of tumor necrosis factor family (TNFRSF19, TNFRSF14, etc). Among the 36 decreased proteins, there were 10 proteins altered by tenfold or more, including ALCAM, VEGF-C, CCL13, etc (Table 2). We first found novel differentially expressed proteins (EREG, IDE, PDGF-D and KNG1) in serum of PCOS patients. The above results provide evidence that some serum proteins in PCOS patients are greatly altered, which may be act as pathogenesis factors of PCOS, and applied as potential biomarkers for PCOS detection.

Analysis of EREG, inhibin $\beta A, I D E$, $P D G F-D$ and KNG1 levels in serum of PCOS patients

Based on the data from above protein

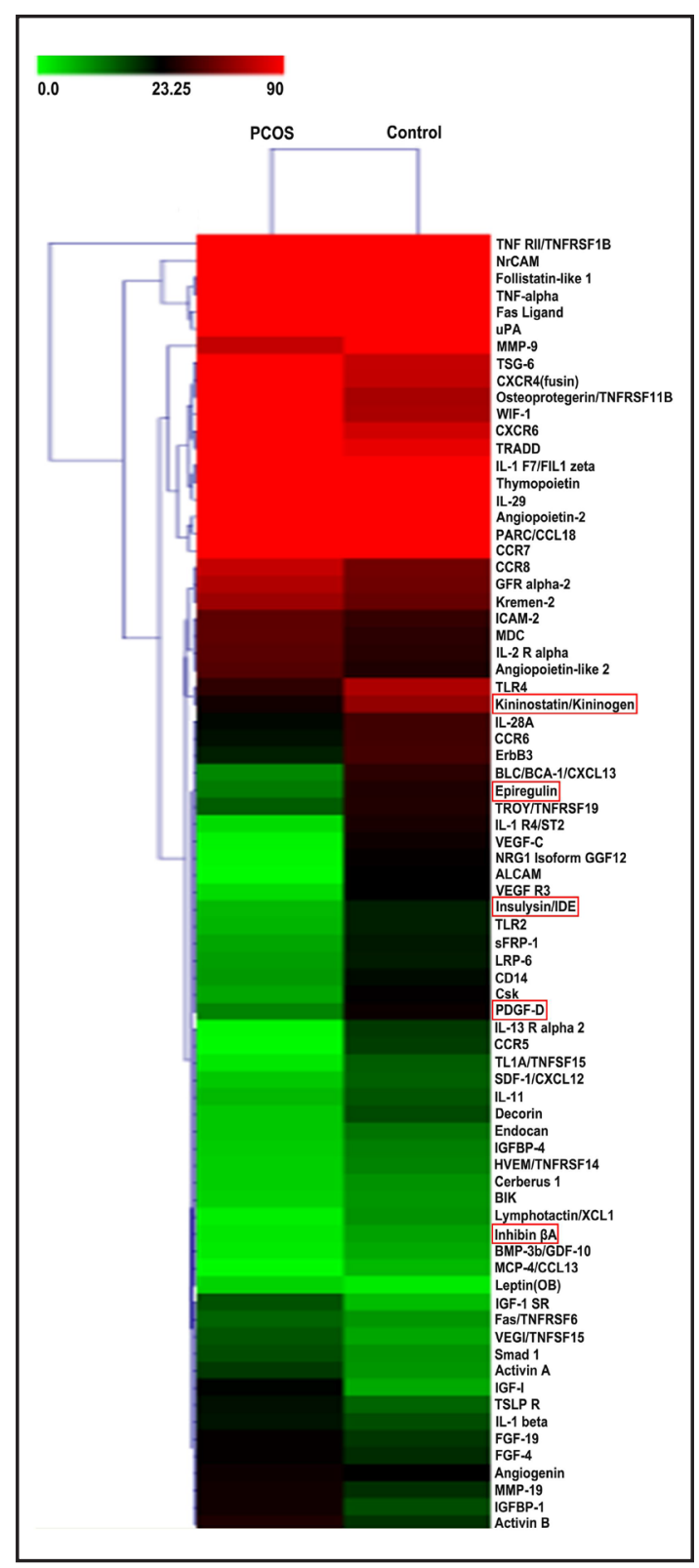

Fig. 1. Protein array of serum proteins from PCOS patients and healthy controls. Clustering diagram of differentially expressed proteins in serum of PCOS patients and healthy controls.

array, we selected five significantly decreased protein molecules, including EREG, inhibin $\beta A$, IDE, PDGF-D and KNG1, to further confirm the alterations in more serum samples of PCOS patients by ELISA and western blot. The serum samples of PCOS (43 cases) with low progesterone level and those from healthy women (30 cases) in childbearing age with normal progesterone level were collected. ELISA assay results showed that in serum of PCOS patients, EREG (Fig. 2A), inhibin $\beta$ A (Fig. 2B), IDE (Fig. 2C), PDGF-D (Fig. 2D) and KNG1 (Fig. $2 \mathrm{E})$ levels were lower as compared to the levels in healthy controls, which were in accordance with the data of protein array. Furthermore, spearman's correlation analysis revealed that the decreased level of the proteins, EREG (Fig. 2F), inhibin $\beta A$ (Fig. 2G), IDE (Fig. 2H), PDGF-D (Fig. 2I) and KNG1 (Fig. 2J), were directly related to the low progesterone value, with the 
correlation coefficient (EREG, $r=0.9359$; inhibin $\beta A, r=0.9516$; IDE, $\quad r=0.9523$; PDGF-D, $r=0.7718$; KNG1, $r=0.9469$ ). The receiver operating characteristic curve (ROC) analysis for each diagnostic index illustrated the discriminative ability between PCOS and healthy women (Fig. $2 \mathrm{~K})$. The area under the ROC curve (AUCs) of EREG (0.926) and inhibin $\beta A$ (0.934) was higher than other three proteins, which revealed that EREG and inhibin $\beta A$ had a higher diagnositic value for PCOS detection. Western blot analysis was also used for the detection of alterations in protein level, and similar changes were observed (Fig. 2L). The results demonstrate that EREG, inhibin $\beta A$, IDE, PDGF-D and KNG1 are decreased in serum of PCOS patients, and may be used as potential biomarkers for the diagnosis.

\section{Upregulation of EREG,} inhibin $\beta A, I D E, P D G F-D$ and KNG1 by progesterone in ovarian granulosa cells

After observing the serological changes of the five proteins in PCOS patients, as well as the correlation with progesterone level, we further determined whether progesterone could regulate these proteins in ovarian granulosa cells. ELISA was performed to assess the level of these five molecules in the culture medium of KGN cells after treatment with different concentrations of progesterone $\left(0,10^{-6}, 10^{-5}\right.$ and $\left.10^{-4} \mathrm{M}\right)$. The results showed that level of EREG (Fig. 3A), inhibin $\beta A$ (Fig. 3B), IDE (Fig. 3C), PDGF-D (Fig. 3D) and KNG1 (Fig. 3E) were increased with elevated progesterone concentrations, and the protein level detected by western blot presented similar changes (Fig. 3K). Moreover, we found that the expression of EREG (Fig. 3F), inhibin $\beta$ A (Fig. 3G), IDE (Fig. 3H), PDGF-D (Fig. 3I) and KNG1 (Fig. 3J) in culture medium were increased, as well as their protein level (Fig. 3L) by stimulation with progesterone for different time periods $(0$, 24,48 and $72 \mathrm{~h}$ ). The results suggest that progesterone induces the synthesis and secretion of EREG, inhibin $\beta$ A, IDE, PDGF-D and KNG1 of ovarian granulosa cells.
Table 1. Increased proteins in PCOS by protein array analysis. *Proteins list with the fold change $\geqq 1.5$ times

\begin{tabular}{|c|c|c|c|}
\hline Type & Name & GenBank accession no. & Fold \\
\hline \multirow[t]{20}{*}{ Growth factor } & $\mathrm{NrCAM}$ & NM_001193584.1 & 1.50 \\
\hline & FGF-4 & NM_002007.2 & 1.56 \\
\hline & Kremen-2 & NM_028416.2 & 1.57 \\
\hline & Thymopoietin & NM_001307975.1 & 1.57 \\
\hline & Follistatin-like 1 & NM_003692.4 & 1.61 \\
\hline & GFR alpha-2 & NM_001495.4 & 1.63 \\
\hline & ICAM-2 & NM_001099789.1 & 1.63 \\
\hline & FGF-19 & NM_005117.2 & 1.65 \\
\hline & TSLP R & NM_00102288.2 & 1.78 \\
\hline & MDC & NM_002990.4 & 1.83 \\
\hline & uPA & NM_001145031.2 & 1.86 \\
\hline & Smad 1 & NM_005900.2 & 1.88 \\
\hline & MMP-19 & NM_001272101.1 & 1.97 \\
\hline & WIF-1 & NM_007191.4 & 2.06 \\
\hline & Acitvin A & NA & 2.16 \\
\hline & IGFBP-1 & NM_000596.2 & 2.34 \\
\hline & Activin B & NA & 2.46 \\
\hline & Leptin (OB) & NM_000230.2 & 2.52 \\
\hline & IGF-1 SR & NA & 3.03 \\
\hline & IGF-1 & NM_001111285.2 & 3.39 \\
\hline \multirow[t]{8}{*}{ Tumor necrosis factor (TNF) } & TNF RII/TNFRSF1B & NM_001066.2 & 1.51 \\
\hline & TNF-alpha & NM_000594.3 & 1.51 \\
\hline & TSG-6 & NM_007115.3 & 1.52 \\
\hline & TRADD & NM_001323552.1 & 1.57 \\
\hline & Fas Ligand & NM_000639.2 & 1.59 \\
\hline & Fas/TNFRSF6 & NM_152871.3 & 1.70 \\
\hline & Osteoprotegerin/ & NM_002546.3 & 1.82 \\
\hline & VEGI/TNFSF15 & NM_001204344.1 & 2.19 \\
\hline \multirow[t]{5}{*}{ Chemokine } & PARC/CCL18 & NM_002988.3 & 1.50 \\
\hline & CXCR4(fusin) & NM_003467.2 & 1.55 \\
\hline & CXCR6 & NM_006564.1 & 1.73 \\
\hline & CCR8 & NM_005201.3 & 1.75 \\
\hline & CCR7 & NM_001301718.1 & 2.04 \\
\hline \multirow[t]{4}{*}{ Interleukin } & IL-1 beta & NM_000576.2 & 1.52 \\
\hline & IL-1 F7/FIL1 zeta & NM_173205.1 & 1.53 \\
\hline & IL-29 & NM_172140.1 & 1.63 \\
\hline & IL-2 R alpha & NM_000417.2 & 1.83 \\
\hline \multirow[t]{3}{*}{ Angiogenesis factor } & Angiogenin & NM_001097577.2 & 1.51 \\
\hline & Angiopoietin-2 & NM_001118888.1 & 1.66 \\
\hline & Angiopoietin-like 2 & NM_012098.2 & 1.94 \\
\hline
\end{tabular}


Promotion of proliferation and inhibition of apoptosis by EREG and inhibin $\beta A$ in ovarian granulosa cells

To determine whether EREG and inhibin $\beta A$ exerted their functions by regulating the proliferation and apoptosis of ovarian granulosa cells, we treated cells with EREG or inhibin $\beta A$, with or without their corresponding specific antibody, respectively. CCK8 assays showed that EREG markedly increased the proliferation of KGN cells compared to the control (Fig. 4A). The addition of the specific EREG antibody alone inhibited cell proliferation, while EREG stimulation restored the proliferation capability to some extent (Fig. 4B). Western blot analysis (Fig. 4C) and immunofluorescent staining results (Fig. 4G) presented an increase of PCNA expression in EREG treated group, which was decreased in the EREG antibody blocking group. Similar results were found when we examined the effect of inhibin $\beta A$ on the proliferation of ovarian granulosa cells (Fig. 4D, 4E, 4F and $\mathrm{H})$. We also investigated the potential roles of EREG and inhibin $\beta A$ in modulating the apoptosis ability of ovarian granulosa cells. The results of
Table 2. Decreased proteins in PCOS by protein array analysis. *Proteins list with the fold change $\leqq 1.5$ times.

\begin{tabular}{|c|c|c|c|}
\hline Type & Name & GenBank accession no. & Fold \\
\hline \multirow[t]{20}{*}{ Growth factor } & NRG1 Isoform GGF2 & NM_013962.2 & 32.26 \\
\hline & BMP-3b/GDF-10 & NM_004962.4 & 3.85 \\
\hline & Epiregulin & NM_001432.2 & 3.50 \\
\hline & inhibin $\beta A$ & NM_002191.3 & 3.00 \\
\hline & Decorin & NM_133503.3 & 2.93 \\
\hline & Insulysin/IDE & NM_004969.3 & 2.81 \\
\hline & Csk & NM_001127190.1 & 2.70 \\
\hline & Kininostatin/Kininogen & NM_001166451.1 & 2.68 \\
\hline & TLR2 & NM_001318796.1 & 2.67 \\
\hline & ErbB3 & NM_001982.3 & 2.62 \\
\hline & sFRP-1 & NM_003012.4 & 2.28 \\
\hline & Endocan & NM_007036.4 & 2.25 \\
\hline & IGFBP-4 & NM_001552.2 & 2.22 \\
\hline & BLK & NM_001197.4 & 2.14 \\
\hline & CD14 & NM_001174105.1 & 2.11 \\
\hline & TLR4 & NM_138557.2 & 2.10 \\
\hline & MMP9 & NM_004994.2 & 2.07 \\
\hline & LRP-6 & NM_002336.2 & 2.06 \\
\hline & Cerberus 1 & NM_005454.2 & 2.04 \\
\hline & UPAR & NM_002659.3 & 1.69 \\
\hline \multirow[t]{3}{*}{ Tumor necrosis factor (TNF) } & TL1A/TNFSF15 & NM_001204344.1 & 6.10 \\
\hline & TROY/TNFRSF19 & NM_018647.3 & 2.49 \\
\hline & HVEM/YNFRSF14 & NM_001297605.1 & 2.33 \\
\hline \multirow[t]{6}{*}{ Chemokine } & CCR5 & NM_000579.3 & 43.38 \\
\hline & MCP-4/CCL13 & NM_005408.2 & 15.63 \\
\hline & Lymphotactin/XCL1 & NM_002995.2 & 6.10 \\
\hline & BLC/BCA-1/CXCL13 & NM_006419.2 & 3.80 \\
\hline & SDF-1/CXCL12 & NM_000609.6 & 2.56 \\
\hline & CCR6 & NM_004367.5 & 2.38 \\
\hline \multirow[t]{4}{*}{ Interleukin } & IL-13 R alpha & NM_000640.2 & 22.22 \\
\hline & IL-1 R4/ST2 & NM_016232.4 & 10.42 \\
\hline & IL-28A & NM_172138.1 & 2.29 \\
\hline & IL-11 & NM_000641.3 & 2.13 \\
\hline \multirow[t]{3}{*}{ Angiogenesis factor } & VEGF-C & NM_005429.4 & 26.32 \\
\hline & VEGF R3 & NM_182925.4 & 6.41 \\
\hline & PDGF-D & NM 025208.4 & 2.32 \\
\hline
\end{tabular}

DAPI staining showed an increased number of apoptotic cells in the specific EREG (Fig. 4I) and inhibin $\beta A$ antibody blocking groups (Fig. 4J); whereas EREG and inhibin $\beta A$ treatment groups presented no detectable changes compared to the control group. Corroboratively, western blot analysis of apoptosis-related proteins demonstrated that the anti-apoptotic protein $\mathrm{Bcl}-2$ was upregulated, and the pro-apoptotic proteins Bax, caspase 8 , and caspase 9 were downregulated after treating with EREG or inhibin $\beta$ A. While blocking EREG and inhibin $\beta A$ with their specific antibodies significantly recovered the levels of apoptosisrelated proteins. Cells treated with EREG and inhibin $\beta A$ specific antibodies significantly downregulated the expression of Bcl-2 and upregulated the levels of Bax, caspase 8 , and caspase 9 (Fig. $4 \mathrm{~K}$ and L). These results demonstrate that EREG and inhibin $\beta A$ promote the proliferation and inhibit the apoptosis of ovarian granulosa cells. 


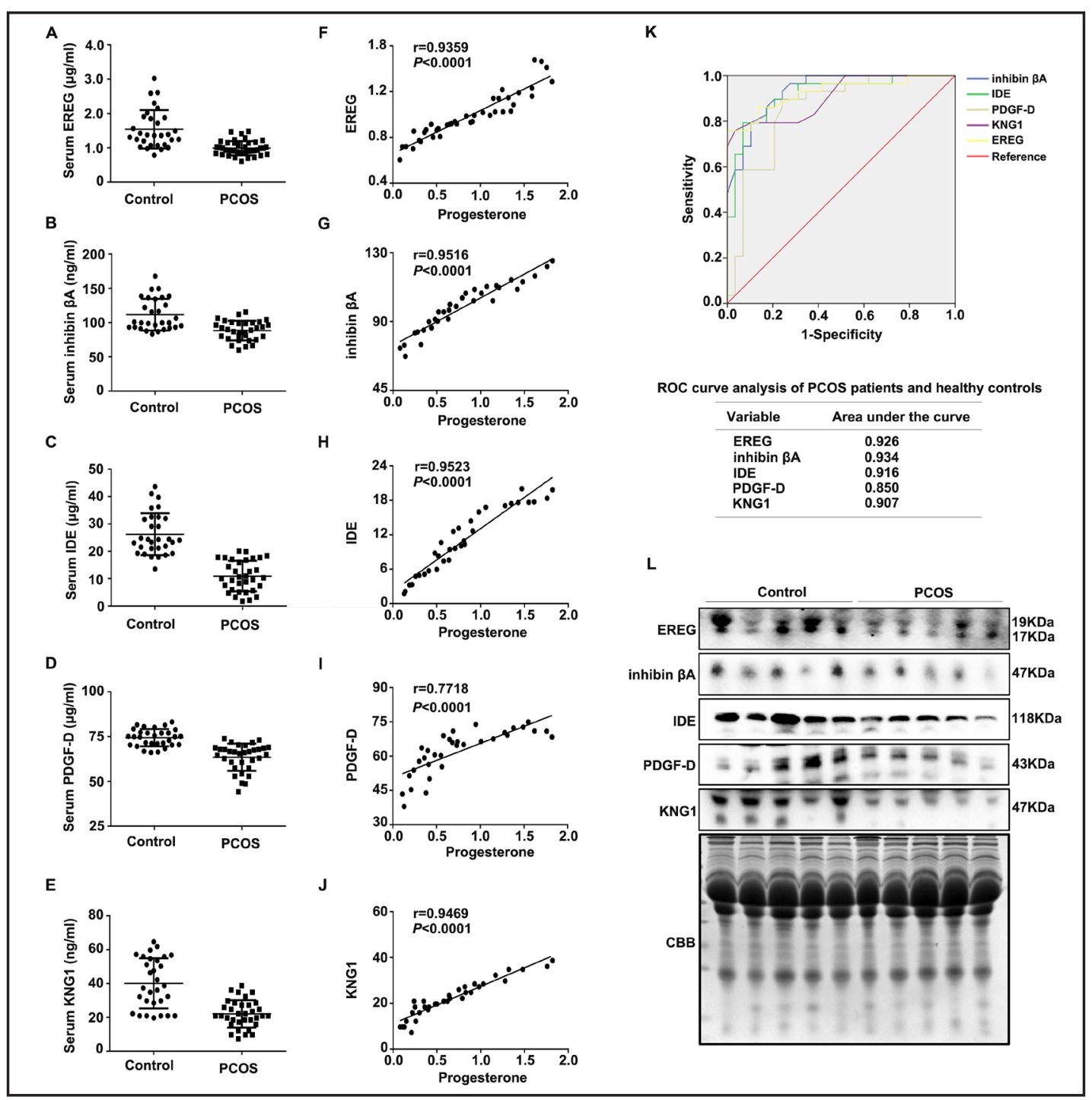

Fig. 2. Analysis of EREG, inhibin $\beta A$, IDE, PDGF-D and KNG1 in serum of PCOS patients. ELISA was used to detect the levels of EREG (A), inhibin $\beta A$ (B), IDE (C), PDGF-D (D) and KNG1 (E) in serum of PCOS patients and healthy controls. The correlation between progesterone and EREG (F), inhibin $\beta A(G)$, IDE (H), PDGF-D (I) and KNG1 (J) in serum of PCOS patients. (K) ROC curve analysis of PCOS diagnostic indexes for EREG, inhibin $\beta$ A, IDE, PDGF-D and KNG1. (L) Western blot analysis of the expression of EREG, inhibin $\beta A$, IDE, PDGF-D and KNG1 in serum of PCOS patients. CBB: Coomassie brilliant blue was used as an equal loading control.

\section{Discussion}

Understanding the general alterations of proteins is not only benefit for elucidating the pathogenesis mechanism of PCOS, but also facilitating for discovering the sensitive and specific biomarkers which are closely associated with diseases. Quantitative analysis of proteins in serum with protein array can supply valuable information for clinical practice, including accurate medical and individualized treatment, which is based on protein screening. Recently, Meltzer et al. examined 500 proteins via protein array in serum samples collected from rectal cancer, and found the expression of OPG was increased which was correlated with the survival of rectal cancer patients after chemotherapy [20]. Jiang et al. analyzed 174 serum proteins of ovarian cancer using protein array screening, and discovered a significant 


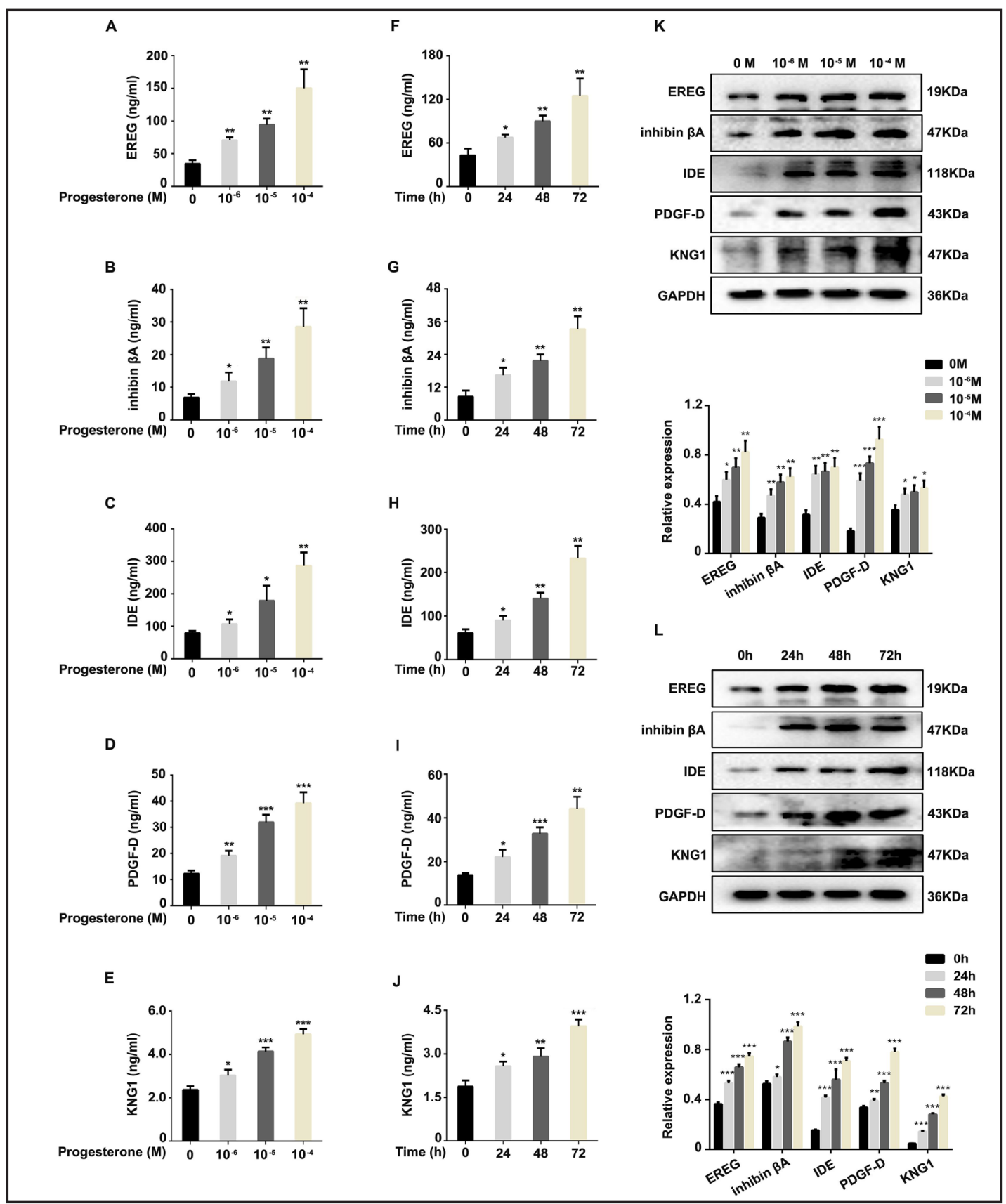

Fig. 3. Upregulation of EREG, inhibin $\beta A$, IDE, PDGF-D and KNG1 by progesterone in KGN cells. ELISA was used to detect the expression of EREG, inhibin $\beta A$, IDE, PDGF-D and KNG1 in the culture medium of KGN cells treated with different concentrations of progesterone $\left(0,10^{-6}, 10^{-5}\right.$ and $\left.10^{-4} \mathrm{M}\right)$ and different times $(0$, 24, 48 and 72 h). Levels of EREG (A), inhibin $\beta$ A (B), IDE (C), PDGF-D (D) and KNG1 (E) detected by ELISA. Expression of EREG (F), inhibin $\beta A(G)$, IDE (H), PDGF-D (I) and KNG1 (J) detected by ELISA. (K) Western blot analysis of EREG, inhibin $\beta A$, IDE, PDGF-D and KNG1 levels after treatment with different concentrations of progesterone. (L) Western blot analysis of EREG, inhibin $\beta A$, IDE, PDGF-D and KNG1 expression after indicated time of progesterone treatment. (Significance is indicated by ${ }^{*} P<0.05,{ }^{* *} P<0.01,{ }^{* * *} P<0.001$ ).

difference of five proteins: MSP-alpha, TIMP-4, PDGF-R alpha, OPG and CA125, which might be regarded as diagnostic biomarkers for ovarian cancer [21]. We, for the first time, compared 507 serum proteins in PCOS patients and healthy controls with protein array, and identified 76 differentially expressed proteins, including 40 increased proteins and 36 


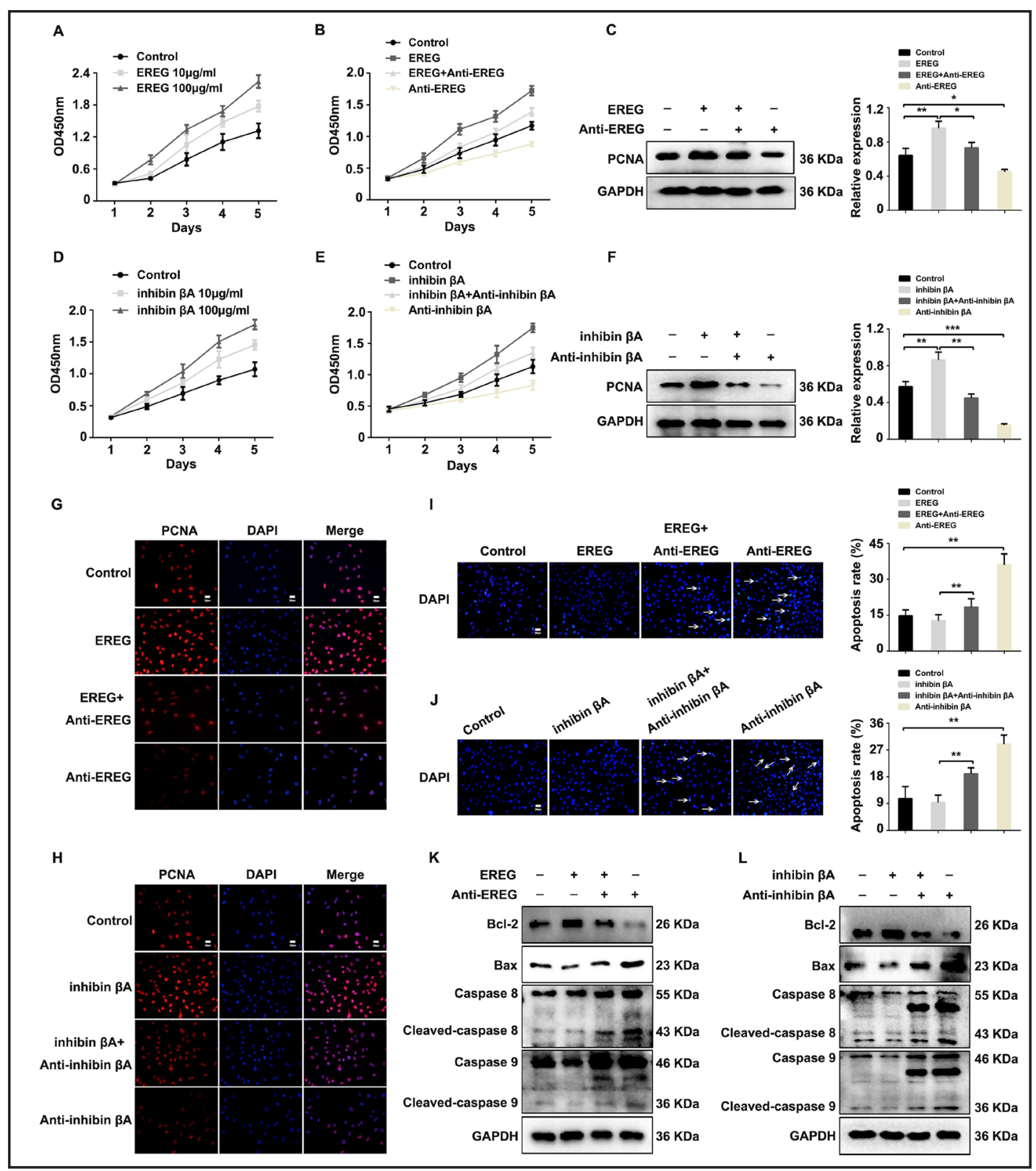

Fig. 4. EREG and inhibin $\beta A$ promoted the proliferation and inhibited the apoptosis of KGN cells. KGN cells were treated with EREG, with or without EREG specific antibody. (A and B) CCK8 assays of cell proliferation. (C) Western blot analysis of PCNA level in KGN cells. (G) Immunofluorescent staining of PCNA in KGN cells. (I) DAPI staining of apoptotic cells. The statistical analysis was shown. (K) Western blot analysis of apoptosis-related protein levels. KGN cells were treated with inhibin $\beta$ A, with or without inhibin $\beta$ A specific antibody. (D and E) CCK8 assays of cell proliferation. (F) Western blot analysis of PCNA level in KGN cells. (H) Immunofluorescent staining of PCNA in KGN cells. (J) DAPI staining of apoptotic cells. The statistical analysis was shown. (L) Western blot analysis of apoptosis-related protein levels. (Significance is indicated by $\left.{ }^{*} P<0.05,{ }^{* *} P<0.01,{ }^{* * *} P<0.001\right)$.

decreased proteins, and 47 kinds of proteins were newly reported. We further confirmed the results by ELISA and western blot, which showed a consistence with the data of protein array. The correlation analysis revealed that the expression of EREG, inhibin $\beta A$, IDE, PDGF-D and KNG1 was positively correlated with progesterone level in serum of patients with PCOS. 
The detection of EREG, inhibin $\beta$ A, IDE, PDGF-D and KNG1 in serum will provide novel and specific potential diagnostic biomarkers for PCOS with low progesterone level.

EREG plays a prominent role in regulating the maturation of follicles and ovarian functions. However, the role of EREG in PCOS-induced infertility has not been characterized. We had demonstrated firstly that the serum level of EREG was decreased in PCOS patients, and progesterone upregulated the expression of EREG. EREG is expressed in ovarian granulosa cells [22], as well as liver progenitor cells. It was reported that the serum level of EREG was increased in patients with liver injury, which promoted the proliferation and DNA synthesis of liver progenitor cells [23]. Moreover, EREG could accelerate the proliferation and differentiation of dental stem cells by activating the MEK/ERK and JNK signaling pathways [24]. Our results showed that the proliferation and apoptosis of ovarian granulosa cells were regulated by EREG. The proliferation ability of ovarian granulosa cells was accelerated with an increased concentration of EREG, and reduced by EREG specific antibody blocking. EREG upregulated the expression of anti-apoptosis protein Bcl-2, and downregulated the expression of pro-apoptosis proteins Bax, caspase 8 and caspase 9, which was reversed by EREG antibody. Therefore, our novel findings emphasize that EREG is upregulated by progesterone, and promotes the proliferation and inhibits the apoptosis of ovarian granulosa cells.

Inhibins, including inhibin A and inhibin $\mathrm{B}$, is produced by ovarian granulosa cells and serves as an essential marker for the development of follicles [25]. Inhibin $\beta A$ is one subunit of inhibin A. Tsigkou A et al. reported that women with PCOS had a higher serum concentration of total inhibin, but lower concentration of dimeric inhibin A than that in healthy control groups [26]. Dafopoulos et al. found that inhibin A was decreased in PCOS patients, and the secretion of inhibin A was sensitive to exogenous FSH [27]. To date, there was no report about the expression of inhibin $\beta A$ in serum of PCOS patients, only total inhibin or dimeric inhibin A. Our screening results showed that the expression of inhibin $\beta A$ differed between PCOS patients and healthy controls. Reduced inhibin $\beta A$ may serve as a potential diagnostic biomarker for PCOS detection. In contrast, Yetim et al. reported that the dimeric inhibin A level in serum of PCOS patients was significantly higher than that in controls [28]. The reasons for the different expression of inhibin $\beta A$ or dimeric inhibin $A$ in patients with PCOS may be as follows. The serum samples from PCOS patients that we analyzed had low progesterone level, which might have caused the decreased level of inhibin $\beta A$ in the present study. Further study was required to investigate the detailed mechanism underlying the decreased serum level of inhibin $\beta A$. We stimulated ovarian granulosa cells with progesterone to examine the functional connection between progesterone and inhibin $\beta A$, and found the expression of inhibin $\beta A$ was increased, which supported that progesterone enhanced the production and secretion of inhibin $\beta A$ in ovarian granulosa cells. Jiang $L$ et al. reported that the ectopic expression of inhibin $\beta A$ in diffuse large B-cell lymphoma (DLBCL) cell lines lead to decreased cell proliferation and increased spontaneous apoptosis, which functioned as a tumor suppressor in DLBCL [29]. In our study, inhibin $\beta A$ promoted cell proliferation, but inhibin $\beta A$ antibody blocking inhibited cell proliferation. DAPI staining and western blot analysis further showed that inhibin $\beta$ A antibody blocking could increase the number of apoptotic cells, as well as apoptosis-related protein levels. Together, our studies suggest that inhibin $\beta A$ stimulates the proliferation and suppresses the apoptosis of ovarian granulosa cells.

As a crucial upstream factor, progesterone has been found to modulate the physiological functions of the ovary via controlling the production and secretion of many molecules. The above results revealed that progesterone promoted the expression of EREG, inhibin $\beta A$, IDE, PDGF-D and KNG1 in ovarian granulosa cells. It had been reported that IDE might be a susceptible gene for PCOS [30]. However, the changes of IDE in serum of PCOS patients remained poorly defined. We first discovered that the serum level of IDE in PCOS patients was notably decreased, and progesterone promoted the expression of IDE in ovarian granulosa cells. These findings demonstrate that IDE may serve as a potential biomarker for the diagnosis of PCOS. The PDGF family members include PDGF-A, PDGF-B, PDGF-C, and

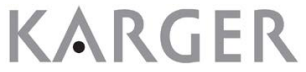


PDGF-D. Kalu et al. detected level of factors including IL-6, TNF-a and PDGF in serum from infertile women and women without endometritis [31]. Scotti et al. found that PDGF-BB and PDGF-DD were significantly decreased in the follicular fluid of PCOS patients [32]. However, no report has examined the changes of PDGF-D in serum of PCOS patients. We screened the changes of serum proteins in PCOS patients, and found that PDGF-D was decreased in patients with low progesterone level. Furthermore, progesterone could upregulate the level of PDGF-D. KNG1 acts as an important regulator of inflammation. It can increase vascular permeability and the release of inflammatory mediators. Kelly et al. reported that chronic inflammation might be involved in the occurrence and development of PCOS [33-34]. Khan et al. found that KNG1 was closely associated with the occurrence and development of reproductive diseases [35]. Indeed, we observed that decreased KNG1 level in serum of PCOS patients, and progesterone promoted the expression of KNG1 in ovarian granulosa cells. Our data demonstrate that changes in serum proteins are related to progesterone in PCOS patients, and progesterone improves ovarian functions by upregulating EREG, inhibin $\beta A$, IDE, PDGF-D and KNG1 expression. The accumulated evidences show that PCOS is one of the major causes leading to infertility [1, 36-37]. Based on the complexity of hormone disorders in PCOS patients, such as high estrogen, high androgen, or low progesterone, as well as the combination of the 2 or 3 of the hormones [38], although the clinical treatment strategy now is mainly by anti-estrogen with clomiphene citrate to induce ovulation and anti-androgen with flutamide to alleviate hyperandrogenism, we propose that progesterone supplementation in combination with routine treatments may improve the therapeutic effect in PCOS patients, and prevent the occurrence of abortion and premature delivery, especially patients with low progesterone level.

In summary, we analyzed the serum proteins of PCOS patients using protein array, and identified five markedly decreased proteins (EREG, inhibin $\beta A$, IDE, PDGF-D and KNG1), which were positively correlated with progesterone. Cytological studies found that progesterone upregulated the expression of these proteins. Furthermore, EREG and inhibin $\beta A$ promoted the proliferation and inhibited the apoptosis of ovarian granulosa cells. Our observations suggest that EREG and inhibin $\beta A$ may be potential serological biomarkers for the detection of PCOS.

\section{Acknowledgements}

This study was supported by the National Natural Science Foundation of China (Grant No.31670810, 31770857 and 81650011).

\section{Disclosure Statement}

We declare that there is no Disclosure Statement regarding the publication of this manuscript.

\section{References}

1 Balen AH, Morley LC, Misso M, Franks S, Legro RS, Wijeyaratne CN, Stener-Victorin E, Fauser BC, Norman RJ, Teede H. The management of anovulatory infertility in women with polycystic ovary syndrome: an analysis of the evidence to support the development of global WHO guidance. Hum Reprod Update 2016;22:687-708.

-2 Lizneva D, Suturina L, Walker W, Brakta S, Gavrilova-Jordan L, Azziz R. Criteria, prevalence, and phenotypes of polycystic ovary syndrome. Fertil Steril 2016;106: 6-15.

3 Carmina E, Guastella E, Longo RA. Advances in the Diagnosis and Treatment of PCOS. Curr Pharm Des 2016;22:5508-5514. 


\section{Cellular Physiology Cell Physiol Biochem 2018;46:2297-2310 \begin{tabular}{l|l} 
DOI: 10.1159/000489619 & $\begin{array}{l}\text { O 2018 The Author(s). Published by S. Karger AG, Basel } \\
\text { www.karger.com/cpb }\end{array}$ \\
\hline
\end{tabular}

4 Lira-Albarrán S, Larrea-Schiavon MF, González L, Durand M, Rangel C, Larrea F. The effects of levonorgestrel on FSH-stimulated primary rat granulosa cell cultures through gene expression profiling are associated to hormone and folliculogenesis processes. Mol Cell Endocrinol 2017;439:337-345.

5 Schwarze JE, Crosby JA, Zegers-Hochschild F. Addition of neither recombinant nor urinary luteinizing hormone was associated with an improvement in the outcome of autologous in vitro fertilization/ intracytoplasmatic sperm injection cycles under regular clinical settings: a multicenter observational analysis. Fertil Steril 2016;106:1714-1717.

6 Gawriluk TR, Ko C, Hong X, Christenson LK, Rucker EB 3rd. Beclin-1 deficiency in the murine ovary results in the reduction of progesterone production to promote preterm labor. Proc Natl Acad Sci U S A 2014;111:E4194-4203.

7 Allen JJ, Herrick SL, Fortune JE. Regulation of steroidogenesis in fetal bovine ovaries: differential effects of LH and FSH. J Mol Endocrinol 2016;57:275-286.

8 Luciano AM, Corbani D, Lodde V, Tessaro I, Franciosi F, Peluso JJ, Modina S. Expression of progesterone receptor membrane component-1 in bovine reproductive system during estrous cycle. Eur J Histochem 2011;55:145-149.

9 Huang S, Pang Y, Yan J, Lin S, Zhao Y, Lei L, Yan L, Li R, Ma C, Qiao J. Fractalkine restores the decreased expression of StAR and progesterone in granulosa cells from patients with polycystic ovary syndrome. Sci Rep 2016;6: 26205-26214.

10 Taraborrelli S. Physiology, production and action of progesterone. Acta Obstet Gynecol Scand 2015;161:816.

11 Livadas S, Boutzios G, Economou F, Alexandraki K, Xyrafis X, Christou M, Zerva A, Karachalios A, Tantalaki E, Diamanti-Kandarakis E. The effect of oral micronized progesterone on hormonal and metabolic parameters in anovulatory patients with polycystic ovary syndrome. Fertil Steril 2010;94:242-246.

-12 Foroozanfard F, Saberi H, Moraveji SA, Bazarganipour F. Pregnancy rate following luteal phase support in Iranian women with polycystic ovarian syndrome. Int J Fertil Steril 2014;8:235-242.

13 Voronina E, Lovasco LA, Gyuris A, Baumgartner RA, Parlow AF, Freiman RN. Ovarian granulosa cell survival and proliferation requires the gonad-selective TFIID subunit TAF4b. Dev Biol 2007;303:715-726.

14 Oróstica L, Astorga I, Plaza-Parrochia F, Vera C, García V, Carvajal R, Gabler F, Romero C, Vega M. Proinflammatory environment and role of TNF-a in endometrial function of obese women having polycystic ovarian syndrome. Int J Obes (Lond) 2016;40:1715-1722.

15 Xia YH, Yao L, Zhang ZX. Correlation between IL-1 $\beta$, IL-1Ra gene polymorphism and occurrence of polycystic ovary syndrome infertility. Asian Pac J Trop Med 2013;6:232-236.

16 Gallinelli A, Ciaccio I, Giannella L, Salvatori M, Marsella T, Volpe A. Correlations between concentrations of interleukin-12 and interleukin-13 and lymphocyte subsets in the follicular fluid of women with and without polycystic ovary syndrome. Fertil Steril 2003;79:1365-1372.

17 Hsieh M, Zamah AM, Conti M. Epidermal growth factor-like growth factors in the follicular fluid: role in oocyte development and maturation. Semin Reprod Med 2009;27:52-61.

18 Blumenfeld Z. Preservation of fertility and ovarian function and minimalization of chemotherapy associated gonadotoxicity and premature ovarian failure: the role of inhibin-A and -B as markers. Mol Cell Endocrinol 2002;187:93-105.

19 Escobar-Morreale HF, Asunción M, Calvo RM, Sancho J, San Millán JL. Receiver operating characteristic analysis of the performance of basal serum hormone profiles for the diagnosis of polycystic ovary syndrome in epidemiological studies.Eur J Endocrinol 2001;145:619-624.

20 Meltzer S, Kalanxhi E, Hektoen HH, Dueland S, Flatmark K, Redalen KR, Ree AH. Systemic release of osteoprotegerin during oxaliplatin-containing induction chemotherapy and favorable systemic outcome of sequential radiotherapy in rectal cancer. Oncotarget 2016;7:34907-34917.

21 Jiang W, Huang R, Duan C, Fu L, Xi Y, Yang Y, Yang WM, Yang D, Yang DH, Huang RP. Identification of five serum protein markers for detection of ovarian cancer by antibody arrays. PLoS One 2013;8:e76795-e76804.

22 Light A, Hammes SR. LH-Induced Steroidogenesis in the Mouse Ovary, but Not Testis, Requires Matrix Metalloproteinase 2- and 9-Mediated Cleavage of Upregulated EGF Receptor Ligands. Biol Reprod 2015;93:1-13. 


\section{Cellular Physiology Cell Physiol Biochem 2018;46:2297-2310

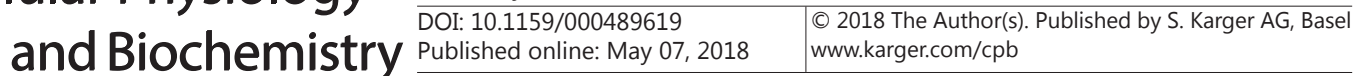 \\ Zheng et al.: Novel Serum Biomarkers for PCOS}

-23 Tomita K, Haga H, Mizuno K, Katsumi T, Sato C, Okumoto K, Nishise Y, Watanabe H, Saito T, Ueno Y. Epiregulin promotes the emergence and proliferation of adult liver progenitor cells. Am J Physiol Gastrointest Liver Physiol 2014;307:G50-57.

-24 Cao Y, Xia DS, Qi SR, Du J, Ma P, Wang SL, Fan ZP. Epiregulin can promote proliferation of stem cells from the dental apical papilla via MEK/Erk and JNK signalling pathways. Cell Prolif 2013;46:447-456.

25 Robertson DM. Inhibins and activins in blood: predictors of female reproductive health? Mol Cell Endocrinol 2012;359:78-84.

-26 Tsigkou A, Luisi S, De Leo V, Patton L, Gambineri A, Reis FM, Pasquali R, Petraglia F.High serum concentration of total inhibin in polycystic ovary syndrome.Fertil Steril 2008;90:1859-1863.

-27 Dafopoulos K, Venetis C, Messini CI, Pournaras S, Anifandis G, Garas A, Messinis IE. Inhibin secretion in women with the polycystic ovary syndrome before and after treatment with progesterone. Reprod Biol Endocrinol 2011;9: 59-963.

28 Yetim A, Yetim Ç, Baş F, Erol OB, Çı̆̆ G, Uçar A, Darendeliler F. Anti-Müllerian Hormone and Inhibin-A, but not Inhibin-B or Insulin-Like Peptide-3, may be Used as Surrogates in the Diagnosis of Polycystic Ovary Syndrome in Adolescents: Preliminary Results. J Clin Res Pediatr Endocrinol 2016;8:288-297.

29 Jiang L, Si T, Yu M, Zeng X, Morse HC 3rd, Lu Y, Ouyang G, Zhou JX.The tumor suppressive role of inhibin $\beta A$ in diffuse large B-cell lymphoma. Leuk Lymphoma 2017;7:1-11.

30 Wang K, You L, Shi Y, Wang L, Zhang M, Chen ZJ. Association of genetic variants of insulin degrading enzyme with metabolic features in women with polycystic ovary syndrome. Fertil Steril 2008;90:378-384.

31 Kalu E, Sumar N, Giannopoulos T, Patel P, Croucher C, Sherriff E, Bansal A. Cytokine profiles in serum and peritoneal fluid from infertile women with and without endometriosis. J Obstet Gynaecol Res 2007;33:490495.

-32 Scotti L, Parborell F, Irusta G, De Zuñiga I, Bisioli C, Pettorossi H, Tesone M, Abramovich D. Platelet-derived growth factor BB and DD and angiopoietin 1 are altered in follicular fluid from polycystic ovary syndrome patients. Mol Reprod Dev 2014;81:748-756.

-33 Kelly CC, Lyall H, Petrie JR, Gould GW, Connell JM, Sattar N. Low grade chronic inflammation in women with polycystic ovarian syndrome. J Clin Endocrinol Metab 2001;86:2953-2455.

34 Zuo T, Zhu M, Xu W, Wang Z, Song H. Iridoids with Genipin Stem Nucleus Inhibit LipopolysaccharideInduced Inflammation and Oxidative Stress by Blocking the NF- $\kappa$ B Pathway in Polycystic Ovary Syndrome. Cell Physiol Biochem 2017;43: 1855-1865.

35 Khan GH, Galazis N, Docheva N, Layfield R, Atiomo W. Overlap of proteomics biomarkers between women with pre-eclampsia and PCOS: a systematic review and biomarker database integration. Hum Reprod 2015;30:133-148.

-36 Pabalan N, Montagna E, Singian E, Tabangay L, Jarjanazi H, Barbosa CP, BiancoB. Associations of Polymorphisms in Anti-Müllerian Hormone (AMH Ile49Ser) and its Type II Receptor (AMHRII -482 A>G) on Reproductive Outcomes and Polycystic Ovary Syndrome: a Systematic Review and Meta-Analysis. Cell Physiol Biochem 2016;39:2249-2261.

-37 Cui N, Wang H, Wang W, Zhang J, Xu Y, Jiang L, Yang A, Hao G. Impact of BodyMass Index on Outcomes of In vitro Fertilization/Intracytoplasmic Sperm InjectionAmong Polycystic Ovarian Syndrome Patients. Cell Physiol Biochem 2016;39:1723-1734.

38 Huang Z\&Yong EL. Ethnic differences: Is there an Asian phenotype for polycystic ovarian syndrome? Best Pract Res Clin Obstet Gynaecol 2016;37:46-55. 